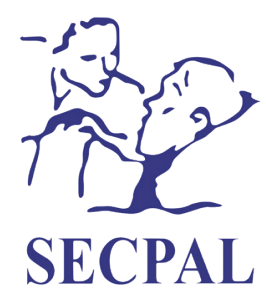

Medicina Paliativa

www.medicinapaliativa.es

REVISIÓN

\title{
Aproximación terapéutica al síndrome de anorexia- caquexia en pacientes con cáncer. Revisión sistemática de la literatura
}

\author{
Ana Ballesteros Abad ${ }^{1}$ y María Nabal Vicuña*2
}

${ }^{1}$ Residente de Medicina de Familia y Comunitaria. ${ }^{2}$ Dra. en Medicina y Cirugía. Responsable del equipo de Cuidados Paliativos. Hospital Universitario Arnau de Vilanova de Lleida. España

Recibido el 18 de septiembre de 2016

Aceptado el 16 de junio de 2017

\section{PALABRAS CLAVE \\ Fatiga, anorexia, caquexia, cáncer, tratamiento.}

\begin{abstract}
Resumen
Objetivo: Realizar una revisión sistemática de la literatura sobre las aproximaciones terapéuticas al síndrome anorexia-caquexia en pacientes oncológicos avanzados.

Material y método: Se realizó una búsqueda de artículos científicos consultando las bases de datos: PubMed, Cochrane, Elsevier, Wiley y Google Scholar, con restricción de fecha 01/01/1990$31 / 12 / 2015$, en los idiomas inglés y español. Las palabras clave utilizadas fueron: "fatigue, anorexia, cachexia, cancer, treatment" y las mismas palabras traducidas al español. En la base de datos PubMed, las ecuaciones de búsqueda utilizadas fueron: "Tumoral cachexia AND treatment" y "Fatigue, anorexia, cachexia AND cancer". Se incluyeron todos los estudios que definieran el síndrome de caquexia tumoral e indicaran al menos una línea de tratamiento para el mismo. Se excluyeron aquellos en los que la caquexia era resultado de una enfermedad terminal diferente al cáncer y en los que el tratamiento había sido enfocado únicamente en la paliación del dolor oncológico. Para seleccionar los artículos se realizó una lectura del título y/o resúmenes de los mismos. En caso de duda, se procedió a la lectura completa del trabajo. Los parámetros analizados fueron: tipo de estudio, criterios de inclusión, tamaño de la muestra, tipo de cáncer, tipos de tratamiento y eficacia de los mismos.

Resultados: en PubMed, con la estrategia de búsqueda "tumoral cachexia AND treatment" encontramos 16 artículos de los cuales un ensayo clínico cumplía los criterios de inclusión. En la misma base de datos, mediante la estrategia de búsqueda: "fatigue, anorexia, cachexia AND cancer" encontramos 106 artículos de los cuales incluimos ocho ensayos clínicos. Como artículo relacionado con la búsqueda anterior incluimos un ensayo clínico más. Además, realizamos una búsqueda de artículos a través de diversas plataformas, e incluimos en la revisión ocho revisiones narrativas y tres ensayos clínicos.
\end{abstract}

\footnotetext{
*Autor para correspondencia:

María Nabal Vicuña

Hospital Universitario Arnau de Vilanova. c/ Rovira Roure, 80. 25198, Lleida, España

Correo electrónico: mnabalv@gmail.com
} 
Conclusiones: 1) La diversidad de estudios revisados imposibilita la realización de un metanálisis. 2) Las medidas terapéuticas de la caquexia se basan en la modulación metabólica. 3) Sigue siendo controvertida que la utilización de suplementos nutricionales mejore el pronóstico del paciente con enfermedad avanzada. 4) La literatura propone diversos tratamientos para el síndrome de caquexia tumoral, siendo en la actualidad el acetato de megestrol el tratamiento de elección. 5) Existen fármacos en desarrollo, como los miméticos de la ghrelina, que ofrecen resultados prometedores.

\section{KEYWORDS}

Fatigue, anorexia, cachexia, cancer, treatment.

\section{Abstract}

Objective: To review what has been published in the scientific literature about the treatment options of the anorexia- cachexia syndrome in cancer patients.

Method: We performed a systematic review of the literature. Databases used were: PubMed, Cochrane, Elsevier, Wiley y Google Scholar, with date restriction 01/01/1990-31/12/2015. Languages were English and Spanish, Key words used were: "fatigue, anorexia, cachexia, cancer in English and Spanish. In PubMed, search strategy were: "Tumoral cachexia AND treatment" and "Fatigue, anorexia, cachexia AND cancer". Articles included had to offer information about any treatment option to the cachexia syndrome. Articles including patients suffering from other illness than cancer were excluded. Papers selection was done base on title and abstract information. In some cases the inclusion was done after reading all the content. Information selected was: type of study, inclusion criteria, simple size, type of cancer, type of treatment and results. Results: In PubMed, based on the search strategy: "tumoral cachexia AND treatment" we found 16 papers but only one was included. Based on the search strategy "fatigue, anorexia, cachexia AND cancer" we found 106 papers but only 8 clinical trials were included. Other related articles were included: 4 clinical trials and 8 narrative reviews.

Conclusion: 1) The methodological differences among the papers reviewed make impossible to develop a methaanalisis. 2) Most of the treatment lines are based on metabolic modulation. 3) It is controversial the use of nutritional supplements to improve prognosis in advanced cancer patient. 4) Among cancer cachexia treatments suggested the megestrol acetate is the election option. 5) New drugs as ghrelin have shown optimistic results.

Ballesteros Abad A, Nabal Vicuña M. Aproximación terapéutica al síndrome de anorexia-caquexia en pacientes con cáncer. Revisión sistemática de la literatura. Med Paliat. 2019;26(1):67-76.

\section{Introducción}

La caquexia es la máxima expresión de desnutrición del paciente oncológico. Se define como un síndrome multifactorial caracterizado por la pérdida de masa muscular, con o sin pérdida de tejido graso, que no responde a soporte nutricional $^{1}$. Se trata de un estado hipercatabólico que supone el consumo energético aumentado en reposo, proteólisis y lipólisis. Analíticamente podemos encontrar hipoproteinemia, hipoalbuminemia, hipertrigliceridemia, anemia, intolerancia a la glucosa o acidosis láctica ${ }^{2}$. Aparecen cambios causados por una respuesta inflamatoria con producción de citoquinas, entre las que destacan el TNF- $\alpha$, IL-1 y IL-6, así como por sustancias producidas por el propio tumor ${ }^{2}$.

La caquexia tumoral es una complicación grave del cáncer, y constituye una causa de morbilidad y mortalidad importante, que puede llegar a afectar a dos tercios de los pacientes oncológicos $^{3}$. La prevalencia global de caquexia tumoral se encuentra en torno al $40 \%$ en los enfermos en el momento del diagnóstico, y al $75 \%$ en fase avanzada de la enfermedad ${ }^{4}$. Esta prevalencia varía según el origen del tumor primario. La supervivencia en pacientes que presentan caquexia es significativamente menor a la de los que no la presentan, pudiendo ser la causa inmediata de muerte del paciente en más de un $20 \%$ de los $\operatorname{casos}^{5}$.
Es fundamental no confundir la caquexia con la pérdida muscular asociada a la edad, la depresión primaria, la malabsorción o el hipertiroidismo, que pueden acontecer al paciente en estadios finales de la vida.

La caquexia se puede clasificar en tres estadios de relevancia clínica ${ }^{5}$, teniendo en cuenta que no necesariamente el paciente tiene que progresar: pre-caquexia, caquexia y caquexia refractaria, cuyas principales características podemos observar en la Tabla I.

Normalmente, la caquexia se asocia a astenia y anorexia, existiendo un desbalance acelerado por la disminución de la ingesta oral por el paciente. Es por ello que se englobaron estas tres identidades para formar el síndrome de asteniaanorexia-caquexia-cáncer ${ }^{6}$.

En cuanto al manejo de la caquexia, se han propuesto diversas estrategias que van desde el tratamiento oncoespecífico del tumor primario hasta el tratamiento de los síntomas relacionados con la falta de apetito (náuseas y vómitos, dolor, estreñimiento, etc.) pasando por intervenciones multimodales que combinan incluso el aumento de la actividad física adaptada a la situación de cada paciente. En lo relativo al tratamiento farmacológico, las drogas eficaces basan su acción en la modulación de citoquinas, hormonas y vías metabólicas. Numerosos tratamientos han sido pro- 
Tabla I. Estadios de la caquexia tumoral

\begin{tabular}{|c|c|c|}
\hline Pre-caquexia & Caquexia & $\begin{array}{l}\text { Caquexia } \\
\text { refractaria }\end{array}$ \\
\hline $\begin{array}{l}\text { Pérdida de peso } \\
<5 \%\end{array}$ & $\begin{array}{l}\text { Pérdida de peso } \\
>5 \% \\
\text { o IMC < } 20 \text { y } \\
\text { pérdida } \\
\text { de peso > } 2 \%\end{array}$ & $\begin{array}{l}\text { Grado de } \\
\text { caquexia } \\
\text { variable }\end{array}$ \\
\hline Anorexia & $\begin{array}{l}\text { Pérdida de peso } \\
>5 \% \\
\text { o sarcopenia y } \\
\text { pérdida } \\
\text { de peso > } 2 \%\end{array}$ & $\begin{array}{l}\text { Cáncer avanzado, } \\
\text { catabolismo } \\
\text { acelerado } \\
\text { y falta de } \\
\text { respuesta a } \\
\text { tratamiento } \\
\text { oncoespecífico }\end{array}$ \\
\hline $\begin{array}{l}\text { Cambios } \\
\text { metabólicos }\end{array}$ & $\begin{array}{l}\text { Disminución de la } \\
\text { ingesta } \\
\text { Mediadores } \\
\text { inflamatorios }\end{array}$ & $\begin{array}{l}\text { Supervivencia } \\
\text { esperada }<3 \\
\text { meses }\end{array}$ \\
\hline
\end{tabular}

Modificada de: Nutr Hosp Supl. 2012;5(1):17-32. puestos para el síndrome de caquexia tumoral, pero solo el acetato de megestrol está aprobado actualmente para esta indicación ${ }^{7}$.

Dada la importancia del problema y sus repercusiones pronósticas, nos planteamos como objetivo realizar una revisión sistemática de la literatura sobre las aproximaciones terapéuticas al síndrome anorexia-caquexia en pacientes oncológicos avanzados.

\section{Material y métodos}

Se realizó una búsqueda de artículos científicos durante diciembre de 2015, consultando las bases de datos PubMed, Cochrane, Elsevier, Wiley y Google Scholar, con restricción de fecha $01 / 01 / 1990-31 / 12 / 2015$, en los idiomas inglés y español.

Las palabras clave utilizadas fueron: "fatigue, anorexia, cachexia, cancer, treatment" y las mismas palabras traducidas al español. En la base de datos PubMed, las ecuaciones de búsqueda utilizadas fueron: "Tumoral cachexia AND treatment" y "Fatigue, anorexia, cachexia AND cancer".

Se incluyeron todos los estudios que definieran el síndrome de caquexia tumoral e indicaran al menos una línea de tratamiento para el mismo. Se excluyeron aquellos en los que la caquexia era resultado de una enfermedad terminal diferente al cáncer y en los que el tratamiento había sido enfocado únicamente en la paliación del dolor oncológico.

Para seleccionar los artículos se realizó una lectura del título y/o resúmenes de los mismos. En caso de duda, se procedió a la lectura completa del trabajo.

Los parámetros analizados fueron: tipo de estudio, criterios de inclusión, tamaño de la muestra, tipo de cáncer, tipos de tratamiento y eficacia de los mismos.

\section{Resultados}

Nuestra búsqueda produjo los siguientes resultados: en PubMed, con la estrategia de búsqueda "tumoral cachexia AND treatment" encontramos 16 artículos de los cuales un ensayo clínico cumplía los criterios de inclusión tras la lectura del título y resumen. En la misma base de datos, mediante la estrategia de búsqueda: "fatigue, anorexia, cachexia AND cancer" encontramos 106 artículos de los cuales, tras la lectura de título y resumen, incluimos ocho ensayos clínicos. Utilizando la herramienta de PubMed "artículo relacionado" incluimos un nuevo ensayo clínico.

Además, realizamos una búsqueda de artículos a través de diversas plataformas, e incluimos en la revisión:

- De la librería Wiley: tres revisiones narrativas y dos ensayos clínicos.

- De la librería Cochrane: una revisión narrativa.

- De Elsevier: una revisión narrativa.

- De Google Schoolar: tres revisiones narrativas y un ensayo clínico.

En las Tablas II y III figuran las características de los ensayos clínicos incluidos en la revisión, así como los tratamientos recogidos y sus resultados.

En lo relativo al contenido, y de manera global, es necesario comentar que la comparación entre los artículos revisados ofrece dificultades debido a la ausencia de consenso en los criterios diagnósticos de caquexia, al tamaño de las muestras, a la duración de los estudios, a la diversidad de tumores que presentaba la población estudiada y a la evolución individual de la enfermedad (Tablas II y III).

Desde 2008, según la comunicación de la Cachexia Consensus Conference, el diagnóstico de caquexia se establece cuando aparece una pérdida de peso mayor o igual al $5 \%$ en los últimos 12 meses, y tres de las siguientes condiciones ${ }^{8}$.

- Disminución de fuerza muscular.

- Astenia.

- Disminución del índice de masa grasa corporal.

- Aumento de marcadores inflamatorios (IL-6).

- Anemia $(\mathrm{Hb}<12 \mathrm{mg} / \mathrm{dl})$.

- Hipoalbuminemia (Alb < 3,2 g/dl).

Los pacientes que formaban parte de los trabajos revisados presentaban cáncer en estadio avanzado, independientemente de que este estuviera siendo tratado específicamente o no, y todos, a criterio de los autores, presentaban caquexia, aunque no cumplían estrictamente los criterios anteriormente citados.

La valoración global del estado nutricional comprende, además de los aspectos antropométricos y valores biológicos ya mencionados, síntomas y signos clínicos. Llama la atención que los autores no comparten los mismos criterios a la hora de establecer el diagnóstico de caquexia, lo cual dificulta la comparación de los estudios y de sus resultados. En los estudios revisados, entre los criterios de inclusión se encuentra la mayoría de edad, el diagnóstico histológico de cáncer en estadio avanzado y la pérdida de peso involuntaria mayor al 5 \% en los últimos meses. Este último criterio lo utilizan como definitorio de caquexia.

En relación con esta pérdida de peso involuntaria, HongSheng Wen ${ }^{9}$ toma de referencia los últimos tres meses; Mantovani ${ }^{10}$ amplía el periodo a cuatro meses y García ${ }^{11}$ y cols. lo hacen hasta seis meses. Destacan los trabajos del equipo de 
Tabla II. Características y resultados de los ensayos clínicos

\begin{tabular}{|c|c|c|c|c|c|c|}
\hline Autor & Año & Tipo ensayo & Muestra & Tipo de cancer & Tratamiento & Resultados \\
\hline Greig CA & 2014 & $\begin{array}{l}\text { Ensayo clínico } \\
\text { fase I/II }\end{array}$ & $\begin{array}{l}\mathrm{n}=13 . \text { Edad } \\
\text { media NC }\end{array}$ & $\begin{array}{l}\text { NC, cáncer } \\
\text { avanzado }\end{array}$ & $\begin{array}{l}\text { Formoterol } 80 \mu \mathrm{g} / \text { día }+ \\
\text { acetato de megestrol } \\
480 \mathrm{mg} / \text { día, } 2 \text { meses }\end{array}$ & $\begin{array}{l}\text { Aumento de masa y/o } \\
\text { función muscular, aumento } \\
\text { de apetito }\end{array}$ \\
\hline $\begin{array}{l}\text { Madeddu } \\
\text { C }\end{array}$ & 2012 & $\begin{array}{l}\text { Ensayo clínico } \\
\text { de no } \\
\text { inferioridad, } \\
\text { fase III, } \\
\text { aleatorizado }\end{array}$ & $\begin{array}{l}\mathrm{n}=60 . \text { Edad } \\
\text { media } 64,5 \\
\text { años }\end{array}$ & $\begin{array}{l}\text { Cabeza y cuello, } \\
\text { pulmón, ovario, } \\
\text { colorrectal, } \\
\text { estómago, } \\
\text { páncreas, esófago, } \\
\text { vías biliares, } \\
\text { hígado }\end{array}$ & $\begin{array}{l}\text { L-carnitina } 4 \mathrm{~g} / \text { día }+ \\
\text { celecoxib } 300 \mathrm{mg} / \text { día } \pm \\
\text { acetato de megestrol } \\
320 \mathrm{mg} / \text { día, } 4 \text { meses }\end{array}$ & $\begin{array}{l}\text { Aumento de la masa } \\
\text { corporal magra, } \\
\text { mejoría de la actividad } \\
\text { física y disminución de la } \\
\text { fatiga. Añadir acetato de } \\
\text { megestrol no demuestra } \\
\text { superioridad en los } \\
\text { resultados }\end{array}$ \\
\hline $\begin{array}{l}\text { Mantovani } \\
\text { G }\end{array}$ & 2010 & $\begin{array}{l}\text { Ensayo clínico } \\
\text { fase III, } \\
\text { aleatorizado }\end{array}$ & $\begin{array}{l}\mathrm{n}=332 \\
\text { Edad media } \\
62 \text { años }\end{array}$ & $\begin{array}{l}\text { Pulmón, mama, } \\
\text { colorrectal, } \\
\text { páncreas, cabeza y } \\
\text { cuello, ovario, } \\
\text { estómago, útero, } \\
\text { riñón, vía biliar, } \\
\text { vejiga, próstata, } \\
\text { esófago, hígado }\end{array}$ & $\begin{array}{l}\text { 1: Medroxiprogesterona } \\
500 \text { mg/día o acetato de } \\
\text { megestrol } 320 \text { mg/día; } \\
\text { 2: EPA 2,2g/día; 3: } \\
\text { L-carnitina } 4 \text { g/día; 4: } \\
\text { talidomida } 200 \text { mg/día; } \\
\text { 5: medroxiprogesterona } \\
500 \text { mg/día o acetato de } \\
\text { megestrol } 320 \text { mg/día + } \\
\text { EPA 2,2 g/día + L- } \\
\text { carnitina } 4 \text { g/día + } \\
\text { talidomida } 200 \text { mg/día, } \\
4 \text { meses }\end{array}$ & $\begin{array}{l}\text { Aumento de la masa } \\
\text { corporal magra, descenso } \\
\text { de REE, disminución de la } \\
\text { fatiga, incremento de } \\
\text { apetito, descenso de IL-6. } \\
\text { El tratamiento más efectivo } \\
\text { fue la combinación de las } 4 \\
\text { drogas, sin encontrar } \\
\text { diferencias entre la } \\
\text { asociación con } \\
\text { medroxiprogesterona o con } \\
\text { acetato de megestrol }\end{array}$ \\
\hline Wen HS & 2012 & $\begin{array}{l}\text { Ensayo clínico } \\
\text { aleatorizado }\end{array}$ & $\begin{array}{l}\mathrm{n}=102 \\
\text { Edad media } \\
62 \text { años }\end{array}$ & $\begin{array}{l}\text { Pulmón, gástrico, } \\
\text { mama, colorrectal, } \\
\text { páncreas, esófago, } \\
\text { hígado }\end{array}$ & $\begin{array}{l}\text { Acetato de megestrol } \\
320 \mathrm{mg} / \text { día } \pm \\
\text { talidomida } 100 \mathrm{mg} / \text { día, } \\
2 \text { meses }\end{array}$ & $\begin{array}{l}\text { Disminución de la fatiga, } \\
\text { aumento de } \\
\text { peso, mejora en la calidad } \\
\text { de vida, aumento de } \\
\text { apetito, descenso de IL- } 6 \text {, } \\
\text { TNF- } \alpha \text {, mejoría GPS y } \\
\text { ECOG, demostrando el } \\
\text { régimen combinado ser } \\
\text { más efectivo que acetato } \\
\text { de megestrol en } \\
\text { monoterapia }\end{array}$ \\
\hline García JM & 2015 & $\begin{array}{l}\text { Ensayo clínico } \\
\text { fase II, } \\
\text { aleatorizado, } \\
\text { doble ciego } \\
\text { controlado } \\
\text { con placebo }\end{array}$ & $\begin{array}{l}\mathrm{n}=82 . \text { Edad } \\
\text { media } 65,3 \\
\text { años }\end{array}$ & $\begin{array}{l}\text { Mama, colorrectal, } \\
\text { pulmón, próstata, } \\
\text { renal, otros }\end{array}$ & $\begin{array}{l}\text { Anamorrelina vs. } \\
\text { placebo, } 4 \text { meses }\end{array}$ & $\begin{array}{l}\text { Aumento de masa corporal } \\
\text { magra con anamorrelina } \\
\text { respecto a descenso de la } \\
\text { misma con placebo }\end{array}$ \\
\hline Jatoi A & 2002 & $\begin{array}{l}\text { Ensayo clínico, } \\
\text { aleatorizado, } \\
\text { doble ciego } \\
\text { controlado } \\
\text { con placebo }\end{array}$ & $\begin{array}{l}n=469 \\
\text { Edad media } \\
66,3 \text { años }\end{array}$ & $\begin{array}{l}\text { Pulmón, } \\
\text { gastrointestinal, } \\
\text { otros }\end{array}$ & $\begin{array}{l}\text { 1: Acetato de megestrol } \\
800 \mathrm{mg} / \text { día ( } 80 \text { días); } \\
\text { 2: dronabinol } 5 \mathrm{mg} / \text { día } \\
\text { ( } 57 \text { días); } 3: \text { acetato de } \\
\text { megestrol } 800 \mathrm{mg} / \text { día } \\
\text { + dronabinol } 5 \mathrm{mg} / \text { día } \\
\text { (74 días) }\end{array}$ & $\begin{array}{l}\text { Aumento de apetito, } \\
\text { aumento de peso, mejora } \\
\text { calidad de vida. Resultados } \\
\text { superiores con acetato de } \\
\text { megestrol que con } \\
\text { dronabinol. La terapia } \\
\text { combinada no demuestra } \\
\text { ser superior al acetato de } \\
\text { megestrol en monoterapia }\end{array}$ \\
\hline $\begin{array}{l}\text { Loprinzi } \\
\text { CL }\end{array}$ & 1999 & $\begin{array}{l}\text { Ensayo clínico } \\
\text { fase III, } \\
\text { aleatorizado }\end{array}$ & $\begin{array}{l}\mathrm{n}=475 \\
\text { Edad media } \\
\mathrm{NC}\end{array}$ & $\begin{array}{l}\text { NC, cáncer } \\
\text { avanzado }\end{array}$ & $\begin{array}{l}\text { 1: Acetato de megestrol } \\
800 \text { mg/día ( } 54 \text { días); } \\
\text { 2: dexametasona } 3 \mathrm{mg} / \text { día } \\
\text { ( } 64 \text { días); } \\
\text { 3: fluoximesterona } \\
20 \text { mg/día ( } 57 \text { días) }\end{array}$ & $\begin{array}{l}\text { Acetato de megestrol } \\
\text { muestra superioridad sobre } \\
\text { fluoximesterona pero } \\
\text { eficacia similar a } \\
\text { dexametasona tanto en la } \\
\text { estimulación del apetito } \\
\text { como en la ganancia de peso }\end{array}$ \\
\hline
\end{tabular}


Tabla II. (Cont). Características y resultados de los ensayos clínicos

\begin{tabular}{|c|c|c|c|c|c|c|}
\hline Autor & Año & Tipo ensayo & Muestra & Tipo de cáncer & Tratamiento & Resultados \\
\hline Macciò A & 2012 & $\begin{array}{l}\text { Ensayo clínico } \\
\text { fase III, } \\
\text { aleatorizado }\end{array}$ & $\begin{array}{l}\mathrm{n}=104 . \\
\text { Edad media } \\
\mathrm{NC}\end{array}$ & $\begin{array}{l}\text { Cáncer } \\
\text { ginecológico }\end{array}$ & $\begin{array}{l}\text { 1: Acetato de } \\
\text { megestrol + L- } \\
\text { carnitina + celecoxib + } \\
\text { antioxidantes; } 2 \text { : } \\
\text { acetato de megestrol, } \\
4 \text { meses }\end{array}$ & $\begin{array}{l}\text { La terapia combinada } \\
\text { muestra } \\
\text { superioridad tanto a nivel } \\
\text { inmunometabólico como } \\
\text { en la calidad de vida }\end{array}$ \\
\hline $\begin{array}{l}\text { Del } \\
\text { Fabbro E }\end{array}$ & 2013 & $\begin{array}{l}\text { Ensayo } \\
\text { clínico, } \\
\text { aleatorizado, } \\
\text { doble ciego, } \\
\text { controlado } \\
\text { con placebo }\end{array}$ & $\begin{array}{l}\mathrm{n}=48 . \\
\text { Edad media } \\
60,5 \text { años }\end{array}$ & $\begin{array}{l}\text { Pulmón, } \\
\text { gastrointestinal }\end{array}$ & $\begin{array}{l}\text { Melatonina } 20 \mathrm{mg} / \text { día } \\
\text { vs. placebo, } 28 \text { días }\end{array}$ & $\begin{array}{l}\text { No hay superioridad } \\
\text { respecto a placebo en la } \\
\text { estimulación del apetito, } \\
\text { aumento de peso o mejora } \\
\text { en la calidad de vida }\end{array}$ \\
\hline Kanat 0 & 2013 & $\begin{array}{l}\text { Ensayo clínico } \\
\text { aleatorizado }\end{array}$ & $\begin{array}{l}\mathrm{n}=62 . \\
\text { Edad media } \\
60,8 \text { años }\end{array}$ & $\begin{array}{l}\text { Pulmón, páncreas, } \\
\text { colorrectal, } \\
\text { estómago, mama, } \\
\text { testículo, } \\
\text { próstata, otros }\end{array}$ & $\begin{array}{l}\text { 1: Acetato de } \\
\text { megestrol + } \\
\text { meloxicam; } 2 \text { : acetato } \\
\text { de megestrol + } \\
\text { meloxicam + EPA; 3: } \\
\text { meloxicam + EPA, } 3 \\
\text { meses }\end{array}$ & $\begin{array}{l}\text { La triple terapia no } \\
\text { muestra } \\
\text { superioridad respecto a la } \\
\text { terapia combinada } \\
\text { meloxicam con acetato de } \\
\text { megestrol o con EPA, } \\
\text { siendo ambas } \\
\text { combinaciones } \\
\text { equivalentes en cuanto a } \\
\text { aumento de peso y } \\
\text { aumento de masa corporal } \\
\text { magra }\end{array}$ \\
\hline Bruera E & 2003 & $\begin{array}{l}\text { Ensayo } \\
\text { clínico, } \\
\text { aleatorizado, } \\
\text { doble ciego, } \\
\text { controlado } \\
\text { con placebo }\end{array}$ & $\begin{array}{l}\mathrm{n}=60 . \\
\text { Edad media } \\
63,8 \text { años }\end{array}$ & $\begin{array}{l}\text { Genitourinario, } \\
\text { mama, pulmón, } \\
\text { hematológico, } \\
\text { cabeza y cuello, } \\
\text { gastrointestinal, } \\
\text { sarcoma }\end{array}$ & $\begin{array}{l}\text { Aceite de pescado (EPA } \\
\text { y DHA) vs. placebo, } 2 \\
\text { semanas }\end{array}$ & $\begin{array}{l}\text { No se encontró mejoría } \\
\text { significativa } \\
\text { en el apetito, estado } \\
\text { nutricional, función física } \\
\text { ni otros síntomas tras la } \\
\text { administración de aceite } \\
\text { de pescado (dosis de } 1,8 \mathrm{~g} \\
\text { de EPA y } 1,2 \mathrm{~g} \text { de DHA } \\
\text { diarios) comparado con } \\
\text { placebo }\end{array}$ \\
\hline Khan ZH & 2002 & $\begin{array}{l}\text { Ensayo clínico } \\
\text { abierto } \\
\text { secuencial }\end{array}$ & $\begin{array}{l}n=11 . \\
\text { Edad media } \\
72,8 \text { años }\end{array}$ & Cáncer esofágico & $\begin{array}{l}\text { Dieta isocalórica, } 2 \\
\text { semanas + talidomida } \\
200 \mathrm{mg} / \text { día, } 2 \text { semanas }\end{array}$ & $\begin{array}{l}\text { Talidomida produce } \\
\text { aumento de } \\
\text { peso y de masa corporal } \\
\text { magra tras dos semanas de } \\
\text { administración diaria } \\
\text { respecto a la disminución } \\
\text { de ambas variables tras } \\
\text { dos semanas de dieta } \\
\text { isocalórica como única } \\
\text { actuación }\end{array}$ \\
\hline $\begin{array}{l}\text { Loprinzi } \\
\mathrm{CL}\end{array}$ & 1990 & $\begin{array}{l}\text { Ensayo } \\
\text { clínico, } \\
\text { aleatorizado, } \\
\text { doble ciego, } \\
\text { controlado } \\
\text { con placebo }\end{array}$ & $\begin{array}{l}\mathrm{n}=133 . \\
\text { Edad media } \\
68 \text { años }\end{array}$ & $\begin{array}{l}\text { Pulmón, } \\
\text { gastrointestinal, } \\
\text { otros }\end{array}$ & $\begin{array}{l}\text { Acetato de megestrol } \\
800 \mathrm{mg} / \text { día vs. } \\
\text { placebo, 8-10 semanas }\end{array}$ & $\begin{array}{l}\text { Acetato de megestrol } \\
\text { demostró estimular el } \\
\text { apetito y aumentar la } \\
\text { ingesta dando lugar a un } \\
\text { aumento de peso } \\
\text { estadísticamente } \\
\text { significativo }\end{array}$ \\
\hline
\end{tabular}


Tabla III. Características de las revisiones bibliográficas

\begin{tabular}{|c|c|c|c|c|}
\hline Autor & Año & Diseño & Tratamiento & Resultados \\
\hline Aoyagi T & 2015 & $\begin{array}{l}\text { Revisión } \\
\text { narrativa }\end{array}$ & $\begin{array}{l}\text { Acetato de megestrol, } \\
\text { medroxiprogesterona, ghrelina, } \\
\text { cannabinoides, antagonistas de la } \\
\text { melanocortina, talidomida, } \\
\text { etanercept, ácidos grasos omega-3, } \\
\text { kampo, cortocoides, AINE, agonistas } \\
\beta 2 \text {-adrenérgicos, quimioterápicos } \\
\text { específicos, medidas no } \\
\text { farmacológicas (dieta, ejercicio físico) }\end{array}$ & $\begin{array}{l}\text { Actualmente todos los tratamientos contra la } \\
\text { caquexia se consideran paliativos, pero nuevos } \\
\text { agentes han incrementado tanto la supervivencia } \\
\text { como la calidad de vida de los pacientes, siendo los } \\
\text { progestágenos los que más evidencia presentan }\end{array}$ \\
\hline Cardona D & 2006 & $\begin{array}{l}\text { Revisión } \\
\text { narrativa }\end{array}$ & $\begin{array}{l}\text { Progestágenos, corticoides, ácidos } \\
\text { grasos omega- 3, cannabinoides, } \\
\text { ciproheptadina, melatonina, } \\
\text { talidomida, anabolizantes, } \\
\text { procinéticos, aminoácidos, } \\
\text { pantoxifilina, sulfato de hidracina }\end{array}$ & $\begin{array}{l}\text { Los progestágenos son los fármacos con mayor nivel } \\
\text { de evidencia en cuanto a estimulación del apetito y } \\
\text { ganancia de peso. Los corticoides tienen efectos } \\
\text { similares durante las primeras cuatro semanas de } \\
\text { tratamiento, después su eficacia disminuye. El resto } \\
\text { de fármacos no muestran evidencias que justifiquen } \\
\text { su uso, excepto los procinéticos que, al aliviar las } \\
\text { náuseas y la saciedad precoz pueden aumentar la } \\
\text { ingesta (no el apetito) }\end{array}$ \\
\hline $\begin{array}{l}\text { Tuca } \\
\text { Rodríguez A }\end{array}$ & 2010 & $\begin{array}{l}\text { Revisión } \\
\text { narrativa }\end{array}$ & $\begin{array}{l}\text { Progestágenos, corticoides, ácidos } \\
\text { grasos omega- 3, talidomida, AINE, } \\
\text { inhibidores COX-2, bortezomib, } \\
\text { procinéticos, ciproheptadina, } \\
\text { sulfato de hidracina, pentoxifilina }\end{array}$ & $\begin{array}{l}\text { El acetato de megestrol tiene eficacia confirmada } \\
\text { por diferentes metanálisis y es el único aprobado } \\
\text { para esta indicación. Los corticoides son eficaces, } \\
\text { pero durante un corto periodo de tiempo. La } \\
\text { asociación de diferentes fármacos activos ha } \\
\text { mostrado mayor eficacia que la monoterapia. La } \\
\text { eficacia de procinéticos, ciproheptadina, sulfato de } \\
\text { hidracina y pentoxifilina no ha podido ser } \\
\text { demostrada en ensayos clínicos controlados }\end{array}$ \\
\hline Vaughan VC & 2012 & $\begin{array}{l}\text { Revisión } \\
\text { narrativa }\end{array}$ & EPA, oxypurinol & $\begin{array}{l}\text { EPA tiene propiedades antitumorales, aumenta la } \\
\text { eficacia de la quimioterapia, disminuye los niveles } \\
\text { de citoquinas inflamatorias, aumenta la masa } \\
\text { corporal magra, incrementa el peso y estimula el } \\
\text { apetito. Se recomienda combinar con actividad } \\
\text { física y otras terapias farmacológicas propuestas } \\
\text { como MA, inhibidores COX-2, etc. La eficacia de } \\
\text { oxypurinol como anticaquéctico todavía no ha sido } \\
\text { comprobada }\end{array}$ \\
\hline $\begin{array}{l}\text { Ingvar } \\
\text { Bosaeus }\end{array}$ & 2007 & $\begin{array}{l}\text { Revisión } \\
\text { narrativa }\end{array}$ & $\begin{array}{l}\text { Corticoides, AINE, anabolizantes, } \\
\text { insulina, eritropoyetina, nutrición } \\
\text { parenteral, suplementos } \\
\text { nutricionales orales }\end{array}$ & $\begin{array}{l}\text { El soporte nutricional como única terapia no } \\
\text { aumenta la supervivencia de los pacientes con } \\
\text { cáncer avanzado. La nutrición parenteral no puede } \\
\text { ser utilizada durante largos periodos de tiempo y } \\
\text { presenta diversas complicaciones. La combinación } \\
\text { de ambas con los fármacos propuestos aumenta la } \\
\text { supervivencia y mejora la calidad de vida }\end{array}$ \\
\hline $\begin{array}{l}\text { Giovanni } \\
\text { Mantovani }\end{array}$ & 2009 & $\begin{array}{l}\text { Revisión } \\
\text { narrativa }\end{array}$ & $\begin{array}{l}\text { Progestágenos, corticoides, ácidos } \\
\text { grasos omega- 3, cannabinoides, } \\
\text { bortezomib, talidomida, ghrelina, } \\
\text { inhibidores COX-2, insulina, BCAA, } \\
\text { oxandrolona, olanzapina }\end{array}$ & $\begin{array}{l}\text { Los progestágenos como el MA y la MP son considerados } \\
\text { el mejor tratamiento posible. Ambos aumentan el peso } \\
\text { corporal y mejoran la calidad de vida del paciente } \\
\text { pero no incrementan la masa corporal magra. Los } \\
\text { corticoides tienen los mismos efectos, pero deben } \\
\text { reservarse para situaciones pre-terminales. Ácidos } \\
\text { grasos omega-3, cannabinoides y bortezomib han } \\
\text { demostrado resultados positivos aunque limitados en } \\
\text { un humanos. Talidomida, ghrelina, inhibidores COX-2, } \\
\text { insulina, BCAA, oxandrolona y olanzapina parecen } \\
\text { demostrar efectividad pero todavía se encuentran en } \\
\text { evaluación clínica }\end{array}$ \\
\hline
\end{tabular}


Tabla III. (Cont.). Características de las revisiones bibliográficas

\begin{tabular}{|c|c|c|c|c|}
\hline Autor & Año & Diseño & Tratamiento & Resultados \\
\hline $\begin{array}{l}\text { Timo D. } \\
\text { Müller }\end{array}$ & 2010 & $\begin{array}{l}\text { Revisión } \\
\text { narrativa }\end{array}$ & Ghrelina & $\begin{array}{l}\text { La infusión continua de ghrelina incrementa el } \\
\text { apetito y disminuye la pérdida de peso. Existe } \\
\text { evidencia de seguridad y tolerancia de ghrelina pero } \\
\text { no existen estudios a largo plazo disponibles por la } \\
\text { potencial implicación del eje ghrelina/GH/IGF-1 en } \\
\text { el crecimiento tumoral y carcinogénesis }\end{array}$ \\
\hline $\begin{array}{l}\text { Takashi } \\
\text { Akamizu }\end{array}$ & 2010 & $\begin{array}{l}\text { Revisión } \\
\text { narrativa }\end{array}$ & Ghrelina & $\begin{array}{l}\text { La administración de ghrelina produce un aumento } \\
\text { de peso y un incremento en la ingesta, } \\
\text { produciéndose un aumento de la masa corporal } \\
\text { magra. Varios ensayos clínicos han demostrado } \\
\text { eficacia y seguridad del fármaco a corto plazo. } \\
\text { Anamorelina en infusión continua también ha } \\
\text { demostrado un aumento de la masa corporal con } \\
\text { tendencia al aumento de la masa magra. Se } \\
\text { requieren estudios a largo plazo que determinen si } \\
\text { ghrelina pudiera estimular el crecimiento tumoral }\end{array}$ \\
\hline
\end{tabular}

Loprinzi $^{12}$ y del equipo de Jatoi ${ }^{13}$, cuyos criterios diagnósticos de caquexia incluyen la pérdida de 5 libras $(2,27 \mathrm{~kg})$ en los últimos dos meses o la ingesta estimada calórica de menos de $20 \mathrm{cal} / \mathrm{kg} /$ día.

Parece oportuno que todos los autores compartan los criterios consensuados en 2008 porque solo si compartimos los criterios diagnósticos tendremos poblaciones comparables y será posible extrapolar los resultados.

El mejor tratamiento para la caquexia tumoral sería el tratamiento curativo del cáncer pero, desafortunadamente, esto no es posible en la mayoría de los $\operatorname{casos}^{6,14}$.

La nutrición como única terapia no ha tenido éxito, ya que no se asocia a mejoría en cuanto a supervivencia, respuesta tumoral, reducción de la toxicidad relacionada con el tratamiento oncoespecífico o de la calidad de vida². Además, aumentar la ingesta en el paciente caquéctico es un reto complicado debido a la anorexia que con frecuencia presenta ${ }^{14}$.

En las normas del American College of Physicians (1989) para pacientes que reciben quimioterapia, no se aconseja proporcionar nutrición parenteral total sistemáticamente. Solo un grupo de pacientes podrían beneficiarse de ello y serían aquellos en los que predomina el componente de ayuno en la etiología de su caquexia ${ }^{2}$. Esto es así ya que se ha constatado que la nutrición parenteral aumenta el número de complicaciones por infección.

En cuanto a la nutrición enteral, existe mayor controversia; según las guías ESPEN ${ }^{15}$ no está recomendado el uso de suplementos nutricionales en el paciente caquéctico. Sin embargo, Boseaus ${ }^{14}$ postula que la combinación de suplementos nutricionales con fármacos moduladores de la respuesta inflamatoria sistémica puede tener efectos beneficiosos en cuanto a supervivencia y calidad de vida.

Respecto a la farmacología utilizada para tratar la caquexia tumoral existe consenso entre la mayoría de autores, que postulan que los progestágenos, como el acetato de megestrol, son el grupo farmacológico mejor considerado para el tratamiento de la caquexia tumoral. Ya en 1990,
Loprinzi y cols. ${ }^{16}$ realizaron un ensayo clínico aleatorizado en el que se incluyeron 133 pacientes con diagnóstico de caquexia tumoral, de los cuales 67 recibieron $800 \mathrm{mg} / \mathrm{dia}$ rios de acetato de megestrol frente a 66 que recibieron placebo. Se encontró una mejoría significativa del apetito y de la ingesta de alimentos en los pacientes tratados con el progestágeno. Además, 11 de los 67 pacientes que recibieron acetato de megestrol experimentaron ganancia ponderal significativa, mientras que solo uno de los pacientes tratados con placebo aumentó su peso. También se constató una disminución de las náuseas y los vómitos estadísticamente significativa en los pacientes tratados con acetato de megestrol.

A día de hoy, el mecanismo de acción del acetato de megestrol no se conoce en profundidad aunque sabemos que está relacionado con la inhibición de citoquinas proinflamatorias (IL-1, IL-6 Y TNF- $\alpha$ ) y con la estimulación del neuropéptido y hipotalámico ${ }^{5}$.

Desde entonces, diferentes metanálisis ${ }^{17}$ han concluido que el acetato de megestrol produce un aumento del apetito y mejora la ingesta, permitiendo aumento de peso no atribuible a retención hídrica. Las dosis analizadas en los diversos estudios han sido muy variables, así los estudios señalan que el acetato de megestrol es eficaz en dosis que oscilan entre $160 \mathrm{mg} /$ día hasta más de $1500 \mathrm{mg} /$ día. Los efectos secundarios de este fármaco son dosis-dependientes, destacando la aparición de edemas, trombosis venosa, rash cutáneo, impotencia, diarrea, prurito y aumento de la glucemia ${ }^{18}$. En este sentido, un estudio posterior realizado por Loprinzi y cols. ${ }^{12}$, comparó la toxicidad del acetato de megestrol respecto a dexametasona. El glucocorticoide demostró una mayor toxicidad que acetato de megestrol, obligando a la interrupción del tratamiento en un $36 \%$ de los pacientes frente al $25 \%$ de los pacientes tratados con acetato de megestrol. La mayor incidencia de trombosis venosa profunda estuvo asociada al tratamiento con megestrol $(5 \text { vs. } 1 \%)^{12}$. 
La dexametaxona es un glucocorticoide utilizado con frecuencia para el tratamiento de la caquexia tumoral ${ }^{2}$. No está claramente establecido el mecanismo de acción, pero se cree que, además de su actividad antiinflamatoria, presenta una actividad orexígena central sobre el hipotálamo ${ }^{17}$. Su principal efecto es la estimulación del apetito y, además, produce una sensación euforizante que proporciona confort al paciente ${ }^{17}$. El beneficio del tratamiento con glucocorticoides es de corta duración y sus efectos secundarios (miopatía, principalmente) son importantes, por ello, no están indicados en pacientes con una esperanza de vida superior a

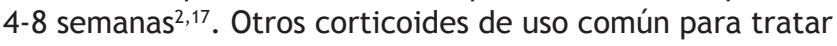
la caquexia tumoral en pacientes con corta expectativa de vida son la prednisona y la metilprednisolona ${ }^{19}$.

En el ensayo clínico realizado por Loprinzi ${ }^{12}$ en 1999, 475 pacientes fueron aleatorizados en tres brazos de tratamiento: acetato de megestrol, dexametasona y fluoximesterona. Los resultados evidenciaron una eficacia similar en cuanto a la estimulación del apetito y ganancia de peso entre acetato de megestrol y dexametasona, y una superioridad de ambas en monoterapia frente a fluoximesterona ${ }^{12}$.

La fluoximesterona es un derivado de la testosterona con potente efecto anabolizante en personas sanas ${ }^{20}$ e indicado en situaciones de catabolismo aumentado, como es la caquexia tumoral. La base fisiopatológica real no se conoce pero se ha comprobado que producen hipertrofia muscular y aumento de la síntesis proteica, con el consiguiente impacto en la masa corporal ${ }^{14,17,20}$. Es por ello que se postuló la hipótesis de su utilidad en los procesos inflamatorios crónicos y en concreto en el contexto de la caquexia de origen tumoral ${ }^{17}$.

Un ensayo clínico fase III presentado en el encuentro ASCO de 2008 comparaba los efectos de oxandrolona, otro fármaco anabolizante androgénico, y acetato de megestrol en 155 pacientes con tumores sólidos y pérdida de peso en el transcurso del tratamiento con quimioterapia ${ }^{6}$. Los resultados concluyeron que los pacientes tratados con el anabolizante experimentaron un aumento de masa corporal magra, una reducción de la grasa corporal y una disminución de la anorexia subjetiva. Actualmente, este fármaco, con resultados prometedores, sigue bajo evaluación clínica 6 .

Otra línea de investigación ha sido la de los ácidos grasos omega-3. El ácido eicosapentaenoico (EPA) y el ácido docosahexaenoico (DHA) son ácidos grasos omega-3 presentes en abundancia en el aceite de pescado ${ }^{3}$, los cuales inhiben la lipólisis y la degradación de la proteína muscular a partir de la inhibición del factor inductor de proteólisis tumoral ${ }^{20}$. Bruera $^{21}$, en 2003, realizó un ensayo clínico en el que 91 pacientes fueron aleatorizados a recibir $180 \mathrm{mg}$ de EPA, 120 $\mathrm{mg}$ de DHA y $1 \mathrm{mg}$ de vitamina $\mathrm{E}$, frente a un grupo control que recibió placebo. Al finalizar el estudio no se encontró mejoría significativa en el apetito, estado nutricional, función física ni otros síntomas tras su administración en monoterapia durante dos semanas, en comparación con placebo ${ }^{21}$.

Una revisión bibliográfica desarrollada por Vaughan en 2012, sobre los ácidos grasos omega-3, reconoce las propiedades antitumorales, así como la capacidad de disminución de citoquinas proinflamatorias que estos poseen. Por tanto, encuentra un papel importante en el tratamiento de la caquexia tumoral de compuestos como el EPA, pero recomienda combinarlos con actividad física y otras terapias farmacológicas, como serían el acetato de megestrol o los inhibidores de la enzima COX-2 para encontrar resultados significativos favorables ${ }^{22}$.
Pero son muchas las sustancias farmacológicas investigadas en el campo de la caquexia.

El dronabinol es un cannabinoide sintético. Su acción se desarrolla en base a su actividad parcialmente agonista en el receptor cannabinoide $\mathrm{CB}_{1}$, ubicado principalmente en el sistema nervioso central, y el receptor $\mathrm{CB}_{2}$, expresado principalmente en las células del sistema inmune ${ }^{2}$.

La marihuana y sus derivados han demostrado la estimulación al apetito, la ganancia de peso en personas sanas $y$, sobre todo, su efecto antiemético en aquellos pacientes con quimioterapia. El mecanismo de la posible ganancia de peso en cancerosos no está claro; puede ser que inhiba la secreción de IL-1 o las prostaglandinas. Los efectos adversos son: euforia, empeoramiento de las funciones cognitivas, vértigos, somnolencia. El dronabinol es el ingrediente activo y responsable de estos efectos. La mayoría de trabajos en la caquexia se han desarrollado en el SIDA².

La talidomida es un fármaco con acción inmunomoduladora al reducir la producción de TNF $\alpha$ y de IL-6. Este es un fármaco bien tolerado, que ha mostrado mejorar significativamente el apetito, las náuseas y la sensación de bienestar ${ }^{2}$. Por sus efectos sedativos, los pacientes experimentan somnolencia diurna transitoria, que desaparece tras 2 o 3 días de tratamiento ${ }^{23}$. Otro efecto secundario frecuente de la talidomida es el estreñimiento, que mejora utilizando laxantes osmóti$\cos ^{23}$. La talidomida puede producir como efecto secundario una neuropatía periférica que se manifiesta con parestesias ${ }^{23}$.

En 2010 se llevaron a cabo dos revisiones bibliográficas sobre el uso de ghrelina ${ }^{24,25}$. La ghrelina es un antagonista de la peptina gástrica cuyas funciones incluyen la regulación de la ingesta, la motilidad gastrointestinal y la secreción ácida gastrointestinal ${ }^{3} \mathrm{y}$, además, posee efecto orexígeno ${ }^{5}$. Por otro lado, la ghrelina estimula la expresión y la liberación de la hormona de crecimiento $(\mathrm{GH})$ e, indirectamente, de IGF-1, (factor inductor del crecimiento), ambas hormonas anabólicas con capacidad de incrementar la masa corporal magra por disminución de la proteólisis ${ }^{23}$. Debido a su mecanismo de acción, se requieren estudios a largo plazo que descarten la implicación del eje ghrelina/GH/IGF-1 en el crecimiento tumoral y carcinogénesis ${ }^{24,25}$.

En 2015, el equipo de García ${ }^{11}$ realizó un ensayo clínico fase II en el que 82 pacientes fueron aleatorizados a recibir anamorelina, una hormona mimética de la ghrelina fisiológica, o placebo durante cuatro meses. Los resultados concluyeron que en los pacientes tratados con anamorelina se produjo un aumento de masa corporal magra respecto al descenso de la misma con placebo ${ }^{26}$.

La melatonina es una hormona endógena producida por la glándula pineal. Su mecanismo de acción se basa en la inhibición del TNF- $\alpha^{19}$. En 2013, Del Fabro ${ }^{26}$ realizó un ensayo clínico en el que 48 pacientes fueron aleatorizados a recibir $20 \mathrm{mg}$ diarios de melatonina frente a placebo durante 28 días. No se demostró superioridad de la terapia con melatonina respecto a placebo en la estimulación del apetito, aumento de peso o mejora en la calidad de vida ${ }^{26}$.

También han sido testados fármacos como la olanzapina ${ }^{6}$ (neuroléptico atípico), ciproheptadina ${ }^{5,18,20}$ (antagonista histamínico y serotoninérgico), pentoxifilina ${ }^{5,20}$ (derivado de la metilxantina con propiedades antinflamatorias por inhibición de fosfodiesterasa y del TNF- $\alpha$ ) o sulfato de hidracina ${ }^{5,20}$ (inhibidor de la gluconeogénesis hepática) que no han confirmado su eficacia mediante ensayos clínicos controlados. 
Actualmente están es estudio fármacos como los antagonistas de la melanocortina ${ }^{3,5}$, los agonistas $\beta-2$ adrenérgicos ${ }^{3,5}$, antagonistas de la IL-65, análogos moduladores de los receptores de andrógenos ${ }^{5}$ o el bortezomib ${ }^{5}$, de los que esperamos resultados en los próximos años.

En lo relativo a la combinación de fármacos, $\mathrm{Greig}^{20}$, en 2014 realizó un ensayo clínico en el que se administró $480 \mathrm{mg}$ al día de acetato de megestrol y $80 \mu \mathrm{g}$ al día de formoterol durante dos meses, encontrando un aumento de masa y función muscular, así como una mejoría en el apetito. En el 2012, Madeddu ${ }^{7}$ propuso la asociación de acetato de megestrol a L-carnitina y celecoxib, no mostrando la triple terapia superioridad respecto a la combinación L-carnitina + celecoxib. En este caso se utilizó L-carnitina por haber demostrado, en estudios con animales, una regulación del TNF- $\alpha$, así como de los niveles de IL-6 séricos. Además, la L-carnitina juega un papel importante en el metabolismo muscular, reduciendo el uso de carbohidratos por parte del músculo durante la realización de ejercicio de baja intensidad. Por otro lado, se considera que es capaz de reducir el estrés oxidativo y la inflamación crónica, ambos involucrados en la fisiopatología de la caquexia ${ }^{7}$. La L-carnitina se combinó con un inhibidor de la COX-2, como es el celecoxib, el cual fue seleccionado por sus propiedades antinflamatorias y anticatabólicas ${ }^{7}$. En ese mismo año, Macciò ${ }^{27}$ desarrolló un ensayo clínico fase III en el que se distribuyeron 104 pacientes en dos brazos. El brazo 1 recibiría acetato de megestrol + L-carnitina + celecoxib + antioxidantes, mientras que el brazo 2 recibiría acetato de megestrol en monoterapia, durante cuatro meses. Los resultados evidenciaron que la terapia combinada mostraba superioridad tanto a nivel inmunometabólico como en lo referente a calidad de vida.

En 2013, el equipo de Kanat ${ }^{28}$ realizó un ensayo clínico en el que un total de 62 pacientes fueron aleatorizados en tres brazos de tratamiento: el brazo 1 recibió acetato de megestrol + meloxicam, el brazo 2 recibió acetato de megestrol + meloxicam + ácido eicosapentaenoico (EPA), y el brazo 3 recibió meloxicam + EPA. La triple terapia no mostró superioridad respecto a la terapia combinada de meloxicam + EPA o de acetato de megestrol + meloxicam y, además, estas dos combinaciones eran equivalentes.

Tanto el ensayo de Madeddu ${ }^{7}$ como el de Kanat ${ }^{28}$ evidencian que la triple terapia progestágeno + suplemento + inhibidor de la enzima COX-2 no obtiene mejores resultados que la terapia combinada suplemento + inhibidor de la enzima COX-2, pero sí es superior al progestágeno en monoterapia, como refleja en ensayo de $\mathrm{Macciò}^{27}$.

Siguiendo la línea de tratamientos combinados con progestágenos, Mantovani ${ }^{10}$ y cols. desarrollaron un ensayo clínico en el que se aleatorizaban 332 pacientes en cinco brazos de tratamiento: medroxiprogesterona o acetato de megestrol, EPA, L- carnitina, talidomida y la combinación de los tres últimos con uno de los dos progestágenos. Los resultados concluyeron que el tratamiento más efectivo fue la combinación de las cuatro drogas, no encontrando diferencias entre la asociación con uno u otro progestágeno ${ }^{10}$. Sin embargo, cuando se combina acetato de megestrol con dronabinol, como planteó el grupo de trabajo encabezado por Jatoi ${ }^{13}$ en 2002, la terapia combinada no demuestra ser superior al acetato de megestrol en monoterapia.

El dronabinol, además de no aportar mejoría en su combinación con acetato de megestrol, es un cannabinoide sintéti- co que presenta alta frecuencia de acontecimientos adversos por alteración de sistema nervioso central, como trastornos de la percepción, alucinaciones, disforia, despersonalización, etc., que son dosis-dependientes y de mayor gravedad en pacientes de edad avanzada ${ }^{2}$.

La talidomida también ha sido combinada con acetato de megestrol en estudios como el desarrollado por el equipo de Wen ${ }^{9}$ en 2010. En este caso, la combinación de estos dos fármacos mostró superioridad en cuanto a disminución de la fatiga, aumento de peso, mejora en la calidad de vida, aumento de apetito y descenso de citoquinas. En el ensayo clínico realizado por Khan ${ }^{23}$ y cols. en 2002, una cohorte de 11 pacientes fue tratada con dieta isocalórica durante dos semanas, controlándose la diferencia de peso y de masa corporal magra al inicio y fin de este periodo. Diez de los 11 pacientes pudieron ser finalmente evaluados. Nueve de ellos experimentaron pérdida de peso y de masa corporal magra respecto a la primera medición. Posteriormente fueron tratados durante dos semanas con $200 \mathrm{mg}$ diarios de talidomida y se observó que solo dos de los 10 pacientes perdieron peso, produciéndose en los ocho restantes un aumento del mismo, así como de la masa corporal magra.

\section{Discusión}

En el presente trabajo hemos querido aproximarnos a lo publicado en torno al tratamiento de la caquexia asociada al cáncer. Nuestra revisión ha sido metódica, pero somos conscientes de que nuestro sistema de búsqueda y acceso a los documentos no está exento de limitaciones. Centrar el problema de la caquexia en cáncer nos ha impedido acceder a publicaciones interesantes de otros ámbitos: investigación básica, nutrición y otras patologías como la enfermedad pulmonar obstructiva crónica o la insuficiencia cardiaca en sus fases avanzadas. Estas pueden ser líneas de futuro.

Una dificultad con la que no contábamos es la ausencia de una definición de consenso previa a 2011, y esta se centra en la compleja interrelación entre la reducción de la ingesta de alimentos, el metabolismo anormal y la pérdida de músculo esquelético. Sin una definición compartida, la comparación de los resultados de los diferentes tratamientos resulta difícil. La definición de consenso incluye un sistema de clasificación en tres etapas: precaquexia, caquexia y caquexia refractaria; pero no todos los trabajos analizados señalan con claridad la fase de caquexia en la que se encuentran los pacientes incluidos. Si tenemos en cuenta que la respuesta de este síndrome varía en función de su gravedad, la ausencia de muestras homogéneas dificulta la correcta evaluación de los diferentes abordajes terapéuticos.

Lo que sí parece claro es que los diferentes fármacos utilizados han intentado responder a diferentes mecanismos fisiopatológicos que parecen relacionarse con la caquexia así: anorexia y baja ingesta, productos inflamatorios y reactantes de fase aguda, interleukinas, etc. Resulta esperanzadora la colaboración entre los departamentos de investigación básica y clínica que seguro nos ofrece frutos interesantes en el futuro.

Nos sorprende el bajo número de trabajos relativos al uso de corticoides como tratamiento de este síndrome cuando durante mucho tiempo fue el fármaco más empleado. Posiblemente nuestra estrategia de búsqueda no ha detectado 
todos los trabajos y algunos hayan quedado excluidos tras la lectura del título y el abstract. También han sido muy escasos los trabajos relativos a tratamientos multimodales combinando medidas farmacológicas y no farmacológicas, cuando parece que la recuperación de la masa muscular se basa tanto en el aporte calórico proteico adecuado como en ejercicio físico adaptado a cada paciente. De cara al futuro nos planteamos revisar las ecuaciones de búsqueda incluyendo términos que incluyan tratamientos no farmacológicos.

Esta revisión, presentada como trabajo de fin de grado, ha sido desarrollada por un único revisor. El hecho de no realizar una doble revisión ciega puede haber facilitado la pérdida de trabajos de interés.

Con todo, podemos concluir que la diversidad de los estudios revisados imposibilita la realización de un metanálisis. Las medidas terapéuticas desarrolladas para el tratamiento de la caquexia se centran en la modulación metabólica. En la actualidad el fármaco de elección es el acetato de megestrol. Fármacos en desarrollo, como los miméticos de la ghrelina, ofrecen resultados prometedores. El uso aislado de los suplementos nutricionales no parece mejorar el pronóstico del paciente en fases avanzadas de caquexia y su uso resulta controvertido.

\section{Bibliografía}

1. Cooper C, Burden ST, Cheng H, Molassiotis A. Understanding and managing cancer-related weight loss and anorexia: insights from a systematic review of qualitative research. J Cachexia Sarcopenia Muscle. 2015;6(1):99-111.

2. Bruera E, Talukdar Rucranath. Capítulo 37: Caquexia. Oncología Clínica. 3. ${ }^{a}$ edición. Barcelona: Elsevier; 2005. p. 749-58.

3. Aoyagi T, Terracina KP, Raza A, Matsubara H, Takabe K. Cancer cachexia, mechanism and treatment. World J Gastrointest Oncol. 2015;7(4):17-29.

4. García-Luna PP, Parejo J, Aliaga A, Pachón J, Serrano P, Pereira JL. Nutrición y cáncer. Nutr Hosp Supl. 2012;5(1):17-32.

5. Tuca A, Calsina-Berna A, González-Barboteo J, Gómez-Batiste X. Caquexia en cáncer. Med Clin. 2010;135(12):568-72.

6. Mantovani G, Madeddu C. Cancer cachexia: medical management. Support Care Cancer. 2010;18(1):1-9.

7. Madeddu C, Dessi M, Panzone F, Serpe R, Antoni G, Cau MC, et al. Randomized phase III clinical trial of a combined treatment with carnitine + celecoxib \pm megestrol acetate for patients with cancer-related anorexia/cachexia syndrome. Clin Nutr. 2012;31(2):176-82.

8. Evans WJ, Morley JE, Argilés J, Bales C, Baracos V, Guttridge D, et al. Cachexia: a new definition. Clin Nutr. 2008;27(6):793-9.

9. Wen HS, Li X, Cao YZ, Zhang CC, Yang F, Shi YM, et al. Clinical studies on the treatment of cancer cachexia with megestrol acetate plus thalidomide. Chemotherapy. 2012;58(6):461-7.

10. Mantovani G. Randomised phase III clinical trial of 5 different arms of treatment on 332 patients with cancer cachexia. Eur Rev Pharmacol Sci. 2013;14(4):292-301.

11. García JM, Boccia RV, Graham CD, Yan Y, Duss EM, Allen S, et al. Anamorelin for patients with cancer cachexia: an integrated analysis of tow phase 2, randomised, placebo-controlled, double-blind trials. Lancet Oncol. 2015;16(1):108-16.
12. Loprinzi CL, Kugler JW, Sloan JA, Mailliard JA, Krook JE, Wilwerding $M B$, et al. Randomized comparison of megestrol acetate versus dexametasona versus fluoxymesterona for the treatment of cancer anorexia-cachexia. J Clin Oncol. 1999;17(10):3299-306.

13. Jatoi A, Windschitl HE, Loprinzi CL, Sloan JA, Dakhil SR, Mailliard JA, et al. Dronabinol versus megestrol acetate versus combination therapy for cancer- associated anorexia. A North Central Cancer Treatment Group study. J Clin Oncol. 2002;20(2):567-73.

14. Bosaeus Ingvar. Nutritional support in multimodal therapy for cancer cachexia. Support Care Cancer. 2008;16:447-51.

15. Arends J, Bodoky G, Bozzetti F, Fearon K, Musariotoli M, Selga $G$, et al. ESPEN guidelines on enteral nutrition: non-surgical oncology. Clin Nutr. 2006;25(2):245-59.

16. Loprinzi CL, Ellison NM, Schaid DJ, Krook JE, Athmann LM, Dose $A M$, et al. Controlled trial of megestrol acetate for the treatment of cancer anorexia and cachexia. J Natl Cancer Inst. 1990;82(13):1127-32.

17. Planas J, Farriols C. Capítulo 4: Síntomas digestivos. Manual de control de síntomas en pacientes con cáncer avanzado y terminal. $2^{\text {a }}$ edición. Madrid: S.A. Aran Ediciones; 2004. p. 133-4.

18. De las Peñas R, Sorribes P. Tratamiento farmacológico de la anorexia y caquexia neoplásicas. [Monografía en internet]. Madrid. Sociedad Española de Oncología Médica. 2004. Disponible en: www.seom.org/seomcms/images/stories/recursos/infopublico/publicaciones/soporteNutricional/pdf/ca p_16.pdf

19. Cardona D. Tratamiento farmacológico de la anorexia-caquexia cancerosa. Nutr Hosp. 2006;21(3):17-26.

20. Creig CA, Johns N, Gray C, MacDonald A, Stephens NA, Skipworth RJ, et al. Phase I/II trial of formoterol fumarate combined with megestrol acetate in cachectic patients with advanced malignancy. Support Care Cancer. 2014; 22(5):1269-75.

21. Bruera E, Strasser F, Palmer JL, Willey J, Calder K, Amyotte G, et al. Effect of fish oil on appetite and other symptoms in patients with advanced cancer and anorexia/cachexia: a doubleblind, placebo-controlled study. J Clin Oncol. 2003;21(1):12934.

22. Vaughan VC, Martin P, Lewandowski PA. Cancer cachexia: impact, mechanisms and emerging treatments. J Cachexia Sarcopenia Muscle. 2013;4(2):95-109.

23. Khan ZH, Simpson EJ, Cole AT, Holt M, McDonald I, Pye D. Oesophageal cancer and cachexia: the effect of short-term treatment with thalidomide on weight loss and lean body mass. Aliment Pharmacol Ther. 2003;17(5):667-82.

24. Akamizu T, Kangawa K. Ghrelin for cachexia. J Cachexia Sarcopenia Muscle. 2010;1:169-76.

25. Müller TD, Pérez-Tilve D, Tong J, Pfluger PT, Tschöp MH. Ghrelin and its potential in the treatment of eating/wasting disorders and cachexia. J Cachexia Sarcopenia Muscle. 2010;1(2):159-67.

26. Del Fabro E, Dev R, Hui D, Palmer L, Bruera E. Effects of melatonin on appetite and other symptoms in patients with advanced cancer and cachexia: a double-blind placebo-controlled trial. J Clin Oncol. 2003;31(10):1271-6.

27. Macciò A, Madeddu C, Gramignano G, Mulas C, Floris C, Sanna $\mathrm{E}$, et al. A randomized phase III clinical trial of a combined treatment for cachexia in patients with gynecological cancers: evaluating the impact on metabolic and inflammatory profiles and quality of life. Gynecol Oncol. 2012;124(3):417-25.

28. Kanat O, Cubukcu E, Avci N, Budak F, Ercan I, Canhoroz M, et al. Comparison of three different treatment modalities in the management of cancer cachexia. Tumori. 2013;99(2):229-33. 